\title{
Mass-symmetry breaking in three-body ions
}

\author{
Vladimir Korobov* and J.-M. Richard \\ Laboratoire de Physique Subatomique et Cosmologie, Université Joseph Fourier - CNRS-IN2P3 \\ 53, avenue des Martyrs, F-38026 Grenoble Cedex, France
}

(Dated: November 21, 2018)

\begin{abstract}
The ground-state energy of three-body ions $\left(M^{+}, M^{+}, m^{-}\right)$evolves when the like-charge constituents are given different masses. The comparison of $\left(m_{1}^{+}, m_{2}^{+}, m^{-}\right)$with the average of $\left(m_{1}^{+}, m_{1}^{+}, m^{-}\right)$and $\left(m_{2}^{+}, m_{2}^{+}, m^{-}\right)$reveals a competition between the symmetric term and the antisymmetric one. The former dominates in the Born-Oppenheimer regime such as the $(p, t, e)$ case, while the latter wins for $\mathrm{H}^{-}$-like systems with two negative light particles surrounding a heavy nucleus. A comparison is also made with the case of baryons in simple quark models with flavour independence.
\end{abstract}

PACS numbers: 36.10.-k,31.15.Ar.31.15.Md

\section{INTRODUCTION}

There are many examples of molecules built out of constituents with identical charge which might acquire different masses when isotopes become involved. For instance, the $\mathrm{H}_{2}$ molecule is seen in several variants such as DT where deuterium and tritium replace the protons. In the field of exotic atoms and molecules, many configurations can be envisaged, such as $\left(p, \mu^{-}, \pi^{-}\right)$where two of the constituents have slightly different masses.

The aim of this paper is to analyze the variation of energy in the simplest case of the ground state of $\mathrm{H}_{2}^{+}$-like configurations. There is already a considerable literature on such systems [1], and tables of very accurate variational energies are available. The mass-dependence of the energy has also been analyzed, and useful approximate formulas have been proposed. Here, we shall restrict ourselves to the specific problem of symmetry breaking.

For consistency, the mass-dependence of the energy $E\left(m_{1}, m_{2}\right)$ of the the $\left(m_{1}^{+}, m_{2}^{+}, m^{-}\right)$ion will be analyzed from the published (or recalculated) values at several neighbouring points, or from an estimate of the first and the second-order perturbation terms calculated with the wave function of a central configuration $\left(M^{+}, M^{+}, m^{-}\right)$.

The behaviour of the $\left(m_{1}, m_{2}, m\right)$ systems as a function of $m_{1}$ and $m_{2}$ for a given third mass $m$ and a given interaction potential was debated some years ago in the framework of confining quark models with flavour independence, that is to say, an interaction that does not change when the mass of any of the quarks is varied, in the same way as the Coulomb potential governs $\mathrm{H}^{-}$, $\mathrm{H}_{2}^{+}$, or $\mathrm{Ps}^{-}$. This note offers the opportunity to make a comparison between the atomic and hadronic cases.

*Permanent address: Joint Institute for Nuclear Research, 141980, Dubna, Russia

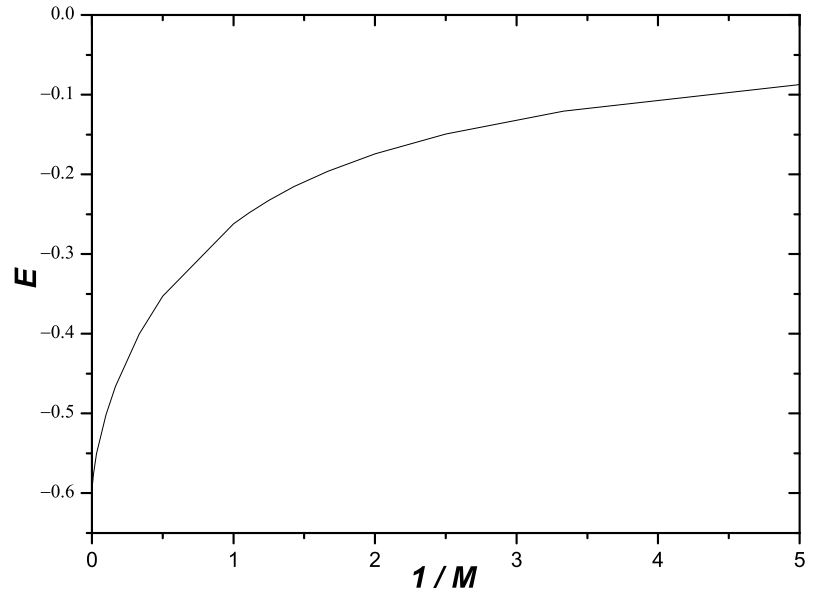

FIG. 1: Ground-state energy of $\left(M^{+}, M^{+}, m^{-}\right)$as a function of $m / M$, for $m=1$.

\section{GENERAL CONSIDERATIONS}

The non-relativistic Hamiltonian is written, with an obvious notation, as

$$
H=\frac{\mathbf{p}_{1}^{2}}{2 m_{1}}+\frac{\mathbf{p}_{2}^{2}}{2 m_{2}}+\frac{\mathbf{p}^{2}}{2 m}+V,
$$

where the potential $V$ is the familiar $V=r_{12}^{-1}-r_{1}^{-1}-r_{2}^{-1}$.

The energy of any level is an increasing function of each inverse mass $m_{i}^{-1}$. This is due to $\mathbf{p}_{i}^{2}$ being positive. For the ground state, the energy is a concave function of any parameter entering the Hamiltonian linearly [2], in particular an inverse mass. The result is true for the ground state of any given angular momentum, or for the sum of the $n$ first levels.

In Fig. 1 the energy of the ground state of the symmetric case, corresponding to the Hamiltonian

$$
H_{\mathrm{S}}(M, m)=\frac{1}{2 M}\left[\mathbf{p}_{1}^{2}+\mathbf{p}_{2}^{2}\right]+\frac{1}{2 m} \mathbf{p}^{2}+V,
$$

is shown as a function of the ratio $x=m / M$, for $m=1$. The monotonic and concave behaviour is clearly seen. 
The values of Fig. 1 can be taken from Ref. [3], but we have preferred to recalculate the energies using a similar variational method employing randomly chosen complex exponents [4]. The regular pattern indicates that a safe interpolation of the binding energy can be done from a few values of the masses.

The concavity property in Fig. 1] shows that, within symmetric configurations, the state $\left(M^{+}, M^{+}, m^{-}\right)$has its energy above the average of $\left(m_{1}^{+}, m_{1}^{+}, m^{-}\right)$and $\left(m_{2}^{+}, m_{2}^{+}, m^{-}\right)$if its inverse mass is taken the exact average, namely,

$$
2 M^{-1}=m_{1}^{-1}+m_{2}^{-1} .
$$

The system of interest is, however, $\left(m_{1}^{+}, m_{2}^{+}, m^{-}\right)$, and it receives a downward shift with respect to its symmetrized version $\left(M^{+}, M^{+}, m^{-}\right)$. Indeed, the Hamiltonian (11) can be rewritten as

$$
H\left(m_{1}, m_{2}, m\right)=H_{\mathrm{S}}(M, m)+\frac{m_{1}^{-1}-m_{2}^{-1}}{2}\left(\mathbf{p}_{1}^{2}-\mathbf{p}_{2}^{2}\right) .
$$

The symmetry-breaking term $\propto\left(m_{1}^{-1}-m_{2}^{-1}\right)$ lowers the ground-state energy, as can be seen by applying the variational principle to (44), with the symmetric ground state of $H_{\mathrm{S}}$ as trial wave function.

In short, when comparing the average of $\left(m_{1}^{+}, m_{1}^{+}, m^{-}\right)$ and $\left(m_{2}^{+}, m_{2}^{+}, m^{-}\right)$to the mixed state $\left(m_{1}^{+}, m_{2}^{+}, m^{-}\right)$, there is a conflict between the concave behaviour in the average inverse mass of the positive particles, and the downward shift due to symmetry breaking.

Starting from the configuration $\left(M^{+}, M^{+}, m^{-}\right)$, one can write the expansions

$$
\begin{aligned}
E\left(m_{i}, m_{i}\right) & =E(M, M)+\lambda_{i} \epsilon_{1, s}+\lambda_{i}^{2} \epsilon_{2, s}+\cdots \\
E\left(m_{1}, m_{2}\right) & =E(M, M)+\bar{\lambda} \epsilon_{1, s}+\bar{\lambda}^{2} \epsilon_{2, s}+\mu^{2} \epsilon_{2, a}+\cdots
\end{aligned}
$$

where the common third mass is omitted and

$$
\begin{aligned}
\lambda_{i} & =\left(2 m_{i}\right)^{-1}-(2 M)^{-1}, \quad \bar{\lambda}=\left(\lambda_{1}+\lambda_{2}\right) / 2, \\
\mu & =\left(4 m_{1}\right)^{-1}-\left(4 m_{2}\right)^{-1},
\end{aligned}
$$

which is reduced to $\bar{\lambda}=0, \lambda_{1}=-\lambda_{2}=\mu$, if $M^{-1}$ is taken as the exact average of inverse masses $m_{1}^{-1}$ and $m_{2}^{-1}$. In the latter case these expansions reduce to

$$
\begin{aligned}
& E\left(m_{1}, m_{2}\right)=E(M, M)+\mu^{2} \epsilon_{2, a}+\cdots \\
& \frac{E\left(m_{1}, m_{1}\right)+E\left(m_{2}, m_{2}\right)}{2}=E(M, M)+\mu^{2} \epsilon_{2, s}+\cdots
\end{aligned}
$$

The coefficients $\epsilon$ can be estimated either by fitting the energies computed for the neighbouring values of the constituent masses, or estimated from the wave function of $\left(M^{+}, M^{+}, m^{-}\right)$, using perturbation theory. The general results listed above translate into $\epsilon_{1, s}>0$ for each level, and $\epsilon_{2, s}<0$ and $\epsilon_{2, a}<0$ for the ground state in any sector of conserved quantum numbers.

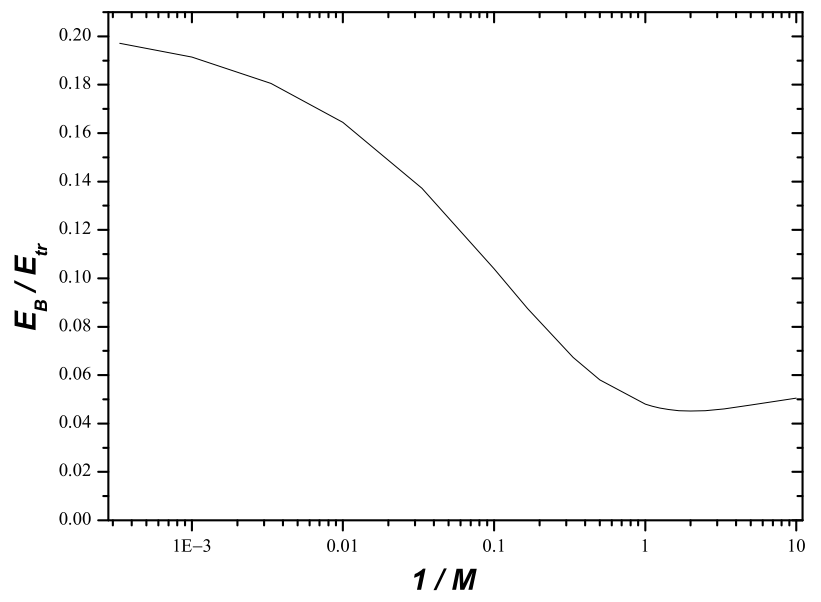

FIG. 2: Binding energy, $E_{B}$, of $\left(M^{+}, M^{+}, m^{-}\right)$as a function of $m / M$ for $m=1$.

\section{RESULTS}

\section{A. Stability}

It can be shown analytically $[5]$ that $\left(M^{+}, M^{+}, m^{-}\right)$is stable against dissociation into $\left(M^{+}, m^{-}\right)$and an isolated $M^{+}$, for any value of the mass ratio $M / m$. The binding energy

$$
E_{B}=E\left(M^{+}, M^{+}, m^{-}\right)-E_{\mathrm{th}}\left(M^{+}, m^{-}\right)
$$

improves dramatically as $M$ increases, in the region where $M>m$. However, $E_{B} / E_{\text {th }}$ is not monotonic in the full range of values of $M / m$. In Fig. 2 , it is seen that the binding energy reaches its minimum at $M \approx 0.5$ and then starts to increase again when the system goes to the $\mathrm{H}^{-}$limit.

\section{B. Concavity vs. symmetry breaking}

In order to analyze the behaviour of $E\left(m_{1}, m_{2}, m\right)$ around the central configuration $E(M, M, m)$ (see Eq. (5)) we introduce the two operators which determine the coefficients of expansion in (5), namely,

$$
V_{s}=\mathbf{p}_{1}^{2}+\mathbf{p}_{2}^{2}, \quad \text { and } \quad V_{a}=\mathbf{p}_{1}^{2}-\mathbf{p}_{2}^{2} .
$$

The perturbation theory gives

$$
\begin{aligned}
& \epsilon_{1, s}=\left\langle\Psi_{0}\left|V_{s}\right| \Psi_{0}\right\rangle, \\
& \epsilon_{2, s}=\left\langle\Psi_{0}\left|V_{s} Q\left(E_{0}-H_{0}\right)^{-1} Q V_{s}\right| \Psi_{0}\right\rangle, \\
& \epsilon_{2, a}=\left\langle\Psi_{0}\left|V_{a}\left(E_{0}-H_{0}\right)^{-1} V_{a}\right| \Psi_{0}\right\rangle,
\end{aligned}
$$

where $Q=I-\left|\Psi_{0}\right\rangle\left\langle\Psi_{0}\right|$ is the projection operator into the subspace orthogonal to $\left|\Psi_{0}\right\rangle$, and $H_{0}$, the symmetric Hamiltonian $H_{S}(M, m)$ with $m=1$. The first order perturbation for $V_{a}$ vanishes. 


\begin{tabular}{rcccc}
\hline \hline$M^{+}$ & $E$ & $\epsilon_{1, s}$ & $\epsilon_{2, s}$ & $\epsilon_{2, a}$ \\
\hline 0.1 & -0.04774837464 & 0.008647 & -0.001567 & -0.009206 \\
0.2 & -0.08729940294 & 0.028959 & -0.009631 & -0.059668 \\
0.3 & -0.12069565567 & 0.055480 & -0.025623 & -0.163192 \\
0.4 & -0.14932813533 & 0.085138 & -0.048893 & -0.314523 \\
0.5 & -0.17418583295 & 0.116148 & -0.078225 & -0.502118 \\
0.6 & -0.19599515999 & 0.147453 & -0.112387 & -0.713757 \\
0.7 & -0.21530357938 & 0.178428 & -0.150301 & -0.938895 \\
0.8 & -0.23253249352 & 0.208710 & -0.191087 & -1.169321 \\
0.9 & -0.24801209057 & 0.238095 & -0.234046 & -1.399056 \\
1 & -0.26200507023 & 0.266477 & -0.278636 & -1.623973 \\
2 & -0.35268735314 & 0.496963 & -0.757118 & -3.356412 \\
3 & -0.40026703884 & 0.658243 & -1.243673 & -4.319080 \\
6 & -0.46608749725 & 0.961169 & -2.754451 & -5.508756 \\
10 & -0.50180015339 & 1.206036 & -5.029963 & -6.049197 \\
30 & -0.55024630859 & 1.851059 & -20.99919 & -6.800187 \\
100 & -0.57644241286 & 2.944942 & -117.3044 & -7.767019 \\
300 & -0.58833372341 & 4.637278 & -595.6470 & -9.343615 \\
1000 & -0.59509329970 & 7.900453 & -3598.177 & -12.52000 \\
3000 & -0.59836899224 & 13.16189 & -18659.60 & -17.73284 \\
\hline \hline
\end{tabular}

TABLE I: The first and second order perturbation for the operators $V_{s}$ and $V_{a}$ for the ground state of the system $\left\{M^{+}, M^{+}, m^{-}\right\}$with $m^{-}=1$.

Results of numerical calculations are presented in Table I. From these data one can get, for instance, an approximate value for the $\mathrm{HD}^{+}$ion ground state, using data for $M=3000$ and Eq. (5),

$$
E\left(m_{p}, m_{d}\right)=-0.597901 \text { a.u. , }
$$

to be compared with the exact solution

$$
E_{\mathrm{HD}^{+}}=-0.597897968 \ldots \text {, }
$$

showing that this rough estimate provides about 6 significant digits for the energy.

From these data one may define approximate bounds for the stability region by solving the equation,

$$
E(M, M)+\mu^{2} \epsilon_{2, a}=-\frac{1}{2}\left(\frac{1}{M}+2 \mu+1\right)^{-1}
$$

with respect to $\mu$. Here we assume that $m_{1}>m_{2}$ and $m=1$. The variation of $\mu$ corresponds to moving along an horizontal line in the triangle of normalized inverse masses 6 . The value of $\mu$ obtained from Eq. (13) for $M=0.1$ is $\mu \approx-0.55$, that corresponds to $m_{1}=0.112$ and $m_{2}=0.0901$, the ratio of these two masses is $m_{1} / m_{2}=1.25$.

The ratio of the symmetric to asymmetric second-order terms (i.e., concavity vs. symmetry breaking) is shown in Fig. 3. In the adiabatic region $M \gg m$, the symmetric term dominates. An inverse hierarchy is observed in the

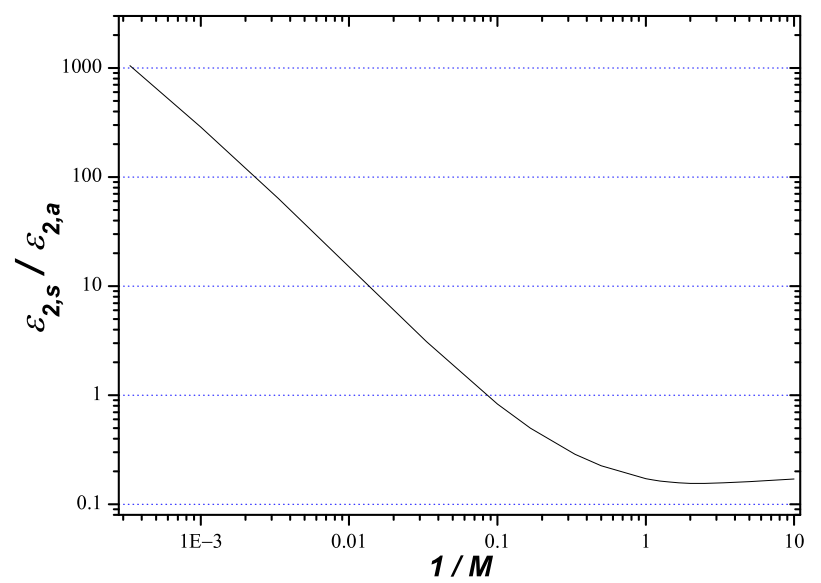

FIG. 3: The ratio of the second order terms (symmetry vs. asymmetry).

\begin{tabular}{ccccc}
\hline \hline$N$ & $\epsilon_{1, s}$ & $\epsilon_{2, s}$ & $N$ & $\epsilon_{2, a}$ \\
\hline 600 & 10.453482 & -8940.3037 & 600 & -15.043224 \\
800 & 10.453482 & -8940.3038 & 800 & -15.043224 \\
\hline \hline
\end{tabular}

TABLE II: The first and second order perturbation for the operators $V_{s}$ and $V_{a}$ for the ground $1 s \sigma_{g}$ state of the $\mathrm{H}_{2}^{+}$.

atomic regime $M \ll m$, with the asymmetric term dominating by about one order of magnitude, implying an inequality

$$
2 E\left(m_{1}, m_{2}\right)<E\left(m_{1}, m_{1}\right)+E\left(m_{2}, m_{2}\right) .
$$

\section{C. $\mathrm{H}_{2}^{+}$"gerade" and "ungerade" ground states}

To get a different insight to the problem, we now compare the concavity and symmetry breaking terms for the $\mathrm{H}_{2}^{+}$molecular ion ground states of different symmetry, starting from the molecule with identical nuclei. The $2 p \sigma_{u}$ (or "ungerade") state is bound by a very shallow potential and lies very close to the threshold. On contrary, the $1 s \sigma_{g}$ potential well supports the existence of 19 vibrational states. Hence it is anticipated that opening the coupling between the "gerade" and "ungerade" states lowers the ground $2 p \sigma_{u}$ state more rapidly than the ground $1 s \sigma_{g}$ state.

Numerical results are presented in Tables II and III. The "ungerade" $\epsilon_{2, a}$ exhibits much slower convergence, since the intermediate state, or the first-order perturbation wave-function, a symmetric function being solution for the $1 s \sigma_{g}$ potential at about zero energy, has multiple nodes that the variational approximation should reproduce with a reasonable accuracy. This makes the numerical calculations more delicate.

The results in the Tables II and III illustrate our conjecture that that the symmetry breaking effect for the case of $2 p \sigma_{u}$ state is much stronger than for the "ger- 


\begin{tabular}{ccccc}
\hline \hline$N$ & $\epsilon_{1, s}$ & $\epsilon_{2, s}$ & $N$ & $\epsilon_{2, a}$ \\
\hline 600 & 1.0497471 & -99.715284 & 1000 & -40051.369 \\
800 & 1.0497471 & -99.725303 & 1400 & -40342.387 \\
1000 & 1.0497471 & -99.725735 & 1800 & -40376.809 \\
\hline \hline
\end{tabular}

TABLE III: The first and second order perturbation for the operators $V_{s}$ and $V_{a}$ for the ground $2 p \sigma_{u}$ state of the $\mathrm{H}_{2}^{+}$.

ade" ground state, while the concavity term is almost negligible.

\section{COMPARISON WITH BARYONS}

In Quantum Chromodynamics (QCD), quarks are coupled to gluons through their colour. The static potential, which serves as first approximation to describe the interaction, is thus independent of the mass of the quarks experiencing this potential. This property, called flavour independence, stimulated several studies on how the spectrum evolves in a given confining potential, as a function of the quark masses. For references, see Refs. 77, 8].

In the case of mesons, once the centre-of-mass motion is removed, the interaction Hamiltonian depends only upon the reduced mass $\mu$, and the inequality [7, 8]

$$
2 E_{2}\left(m_{1}, m_{2}\right) \geq E_{2}\left(m_{1}, m_{1}\right)+E_{2}\left(m_{2}, m_{2}\right)
$$

holds whatever flavour-independent potential $V\left(r_{12}\right)$ is assumed.

In the case of baryons, it was always found for plausible flavour-independent potentials [9], that

$2 E_{3}\left(m_{1}, m_{2}, m\right) \geq E_{3}\left(m_{1}, m_{1}, m\right)+E_{3}\left(m_{2}, m_{2}, m\right)$.

The approximate equality observed for experimental baryon masses (the contribution of constituent masses is identical on both sides) is due to a cancelation of this concave behaviour with the convex behaviour of hyperfine corrections.
It was even conjectured that Eq. (16) might be generally true, though resisting proof. Then Lieb found 10] counterexamples, which were further extended in [8, 11]. Lieb also found wide classes of potentials for which the inequality (16) is rigorously proven. Note that the quark problem does not include the case of $\mathrm{H}_{2}^{+}$-like ions, since the interquark potential $V=\sum v\left(r_{i j}\right)$ is assumed to be fully symmetric.

\section{CONCLUSION}

In this article, we have studied the effect of breaking the $m_{1}=m_{2}$ symmetry in the ground state $\left(m_{1}^{+}, m_{2}^{+}, m^{-}\right)$. Two regimes are identified. For $m_{1}$ and $m_{2}$, much heavier than the negatively charged particle of mass $m$, the heavy particles experience an effective twobody potential. Then the dynamics depends mainly on the sum of inverse masses, a property that becomes exact in the case of two-body systems, or in the case of mesons in a flavour-independent quark-antiquark potential. In this situation, the inequality (16) is observed. Now, for $m_{1}$ and $m_{2}$ of the order of $m$ or smaller, in the $\mathrm{H}^{-}$limit, the asymmetric term $\left(\mathbf{p}_{1}^{2}-\mathbf{p}_{2}^{2}\right)$ plays a more important role, and the reversed inequality is observed.

For the adiabatic systems, which have both symmetric and antisymmetric bound states, the symmetry breaking effect is much stronger for the upper antisymmetric state, and is the dominant contribution, while the concavity term $\left(\mathbf{p}_{1}^{2}+\mathbf{p}_{2}^{2}\right)$ is rather negligible.

We intend to study the case of excited states, and more complicated structures, such as hydrogen-molecule-like states $\left(m_{1}^{+}, m_{2}^{+}, m^{-}, m^{-}\right)$, and to extend the results of our present work to the higher order perturbation terms.

\section{Acknowledgments}

We thank P. Valiron for stimulating discussion and M. Asghar for comments on the manuscript.
[1] E.A.G. Armour and W. Byers Brown, Accounts of Chemical Research, 26168 (1993), and references therein.

[2] W. Thirring, A Course in Mathematical Physics, Vol. 3: Quantum Mechanics of Atoms and Molecules (Springer Verlag, New-York, 1979).

[3] A.M. Frolov and D.M. Bishop, Phys. Rev. A 45, 6236 (1992).

[4] V.I. Korobov, Phys. Rev. A 61, 064503 (2000).

[5] R.N. Hill, J. Math. Phys. 18, 2316 (1977).

[6] A. Martin, J.-M. Richard, and Tai Tsun Wu, Phys. Rev. A 46, 3697 (1992).
[7] H. Grosse and A. Martin, Particle physics and the Schrödinger equation, Cambridge, UK: Univ. Pr. (1997) 167 p. (Cambridge monographs on particle physics, nuclear physics and cosmology, vol. \#6).

[8] S. Nussinov and M. A. Lampert, Phys. Rept. 362 (2002) 193 arXiv:hep-ph/9911532.

[9] J.-M. Richard and P. Taxil, Ann. Phys. (N.Y.) 150 (1983) 267.

[10] E. H. Lieb, Phys. Rev. Lett. 54, 1987 (1985).

[11] A. Martin, J. M. Richard and P. Taxil, Phys. Lett. B 176, 224 (1986). 